\title{
The Numerical Chromosomal Abnormalities in Mentally Retarded Children within Age Group of 1-16 Years
}

\author{
Dr. Parinda Barot ${ }^{1 *}$, Dr. Saiyad SS ${ }^{2}$
}

${ }^{1}$ Department of Anatomy, P.D.U. Medical College, Rajkot, Gujarat, India

${ }^{2}$ Prof. \& Head, DDU Medical College, Nadiad, Gujarat, India

DOI: $10.36348 /$ sijap.2020.v03i08.001

| Received: 26.07.2020 | Accepted: 03.08.2020 | Published: 09.08.2020

*Corresponding author: Dr. Parinda Barot

\section{Abstract}

The cytogenic study included 20 mentally retarded children within the age group of 1 to 16 years, were evaluated for numerical chromosomal abnormalities. Out of 20 subjects, the first 11 samples were taken from the children of the school of the Mentally Retarded People, university road, Rajkot and the rest 09 samples were collected from the patients visited to Psychiatric OPD at the Government Civil Hospital, Rajkot. $15 \%$ female subject while $85 \%$ male subjects were evaluated for the present study. The selection criteria were children of the age between 1 and 16 years and the confirmation of mental retardation. The severity of category of mental retardation has not been focused. Chromosome preparation from peripheral blood cultures was done using standard techniques and cytogenic analysis was performed using GTG-Banding technique. 10\% Karyotype were observed with numerical chromosomal abnormalities and that is Trisomy of $21^{\text {st }}$ pair of chromosome. The study concluded that chromosomal abnormalities are thought to be the most common cause of mental retardation. However, apart from a few selected types with typical aneuploidy, like Down's syndrome, the frequency of detectable chromosomal abnormalities in association with idiopathic mental retardation is very low.

Keywords: Numerical Chromosomal abnormalities, Mentally Retarded Children.

Copyright @ 2020: This is an open-access article distributed under the terms of the Creative Commons Attribution license which permits unrestricted use, distribution, and reproduction in any medium for non-commercial use (NonCommercial, or CC-BY-NC) provided the original author and source are credited.

\section{INTRODUCTION}

Genetics is the science of heredity. Human genetics deals with the inherited characters - physical \& mental, normal as well as abnormal - in an individual, a family, a race or a population. It is concerned with the ways in which these characters are transmitted from generation to generation.

Organelles found in pairs in the eukaryotic nuclei, have a species-specific number, which for a man is 46 or 23 pairs. Twenty- two of these are autosomes and the remaining pair, the sex chromosomes, are designated $\mathrm{X}$ and $\mathrm{Y}$, because of their dissimilarity and their role in sex determination.

During mitosis or in the second division of meiosis, these errors in the chromosome number may result from the failure of sister chromatids to separate correctly at anaphase. Similarly, during the first division of meiosis, paired homologous chromosomes may fail to move separately at the opposite poles during anaphase.
Autosomal trisomies occur relatively more frequently; these involve chromosomes 13, 18 \& 21, giving rise to Patau, Edwards and Down syndrome respectively. Trisomy 21 is the most common being associated with a birth frequency risk of 1 in 700 population.

Mental Retardation refers to substantial limitations in present functioning. It is characterised by significantly sub average intellectual functioning, existing concurrently with related limitations in applicable adaptive skills. There are many causes of mental retardation. The most common causes are Genetic diseases. Problems during pregnancy, perinatal problems and the other health diseases like whooping cough, the measles, or meningitis. Mental retardation can also be caused by extreme malnutrition or being exposed to poisons like lead or mercury.

In the present study numerical chromosomal abnormalities have been focused, other chromosomal abnormalities are structural. 
Intellectual functioning level is defined by standardised tests that measure the ability to reason in terms of mental age (intelligence quotient or IQ). Mental retardation is defined as an IQ score below 7075[1]. Severity of MR: This was categorised according to the World Health Organisation classification and DSM-IV criteria: profound ( $\mathrm{IQ}=0-20)$; severe $(\mathrm{IQ}=21$ $35)$; moderate $(\mathrm{IQ}=36-50)$; mild $(\mathrm{IQ}=51-70)$; borderline $(\mathrm{IQ}=71-85)$.

Children who are mentally retarded reach developmental milestones significantly later than expected, if at all. If retardation is caused by chromosomal or other genetic disorders, it is often apparent from infancy. Mental retardation affects $3 \%$ of the population, yet a diagnosis is obtained in only about a third of cases. Our poor understanding of its origin hampers the provision of effective treatment and preventive regimens, and remains a major challenge for medical practice.

To date, there have been numerous studies regarding the aetiology of mental retardation. The results revealed much variability. Van Karnebeek CDM, Scheper FY, Abeling NG [2] noted wide range of reported frequencies of chromosomal abnormalities causing mental retardation $-2 \%$ to $50 \%$ depending on the variation in the study design among published reports. Shevell M, Ashwal S, Donley D [3] reported the range of chromosomal abnormalities found on routine cytogenic analysis to be $2.93 \%$ to $11.6 \%$ with a median of 3\%. Samantha J L Knight, Regina Regan, Alison Nicod, Sharon W Horsley, Lundal Kearney, Tesan Homfray, Robin M Winter, Patrick Bolton, Jonathan Flint [4], used fluorescence in situ hybridization (FISH) test was to examine the integrity of chromosome ends. Subtle chromosomal abnormalities occurred with a frequency of $7.4 \%$ in the children with moderate to severe mental retardation, and of $0.5 \%$ in the children woth mild mental retardation. Samikshan Dutta, Jyoti Shaw, Swagata Sinha, Kanchan Mukhopadhyay [5] have investigated chromosomal abnormalities in female MR subjects $(n=150)$ by high resolution GTG banding. Of them 30 cases were diagnosed as Downs's syndrome.

Analysis of the relationship between yield of chromosome studies and clinical settings was hampered by uneven distribution of studies among the different settings. Most studies were performed in institutions, which on average will comprise more severely retarded patients than schools and population surveys. The median frequency of anomalies was higher in individuals with moderate to severe MR than those with borderline or mild MR.

\section{MATERIALS AND METHODS}

Out of 20 subjects, the first 11 samples were taken from the children of the Society of Mentally Retarded People, University road, Rajkot, and the rest 09 samples were taken from the patients of Psychiatric OPD at the Government civil hospital, Rajkot. Out of 20 patients 03 were females and 17 were male patients. The selection criteria were the children of the age between 1 and 16 years and the confirmation of mental retardation. The severity of category of mental retardation has not been focused.

In the present study peripheral venous blood is used. With the aseptic precautions $2 \mathrm{ml}$ of venous blood was collected by venepuncture, in a heparinised syringe. $0.7 \mathrm{ml}$ of this heparinised blood was added to $5 \mathrm{ml}$ of RPMI medium containing $0.2 \mathrm{ml}$ phytoheamaglutinin, $1 \mathrm{ml}$ of human $\mathrm{AB}$ positive serum and antibiotics, in a pre-autoclaved culture bottle, gently shaken and incubated at $37^{\circ} \mathrm{C}$. After 69 hours of incubation, $10 \mu \mathrm{l} / \mathrm{ml}$ colchicines was added and incubated further for 30mins. Followed by hypotonic treatment with KCL and again incubated for 1 hour. After three successive changes with fixative, cells suspended in about half $\mathrm{ml}$ of fixative and are dropped from height on chilled slides. Ageing the slides in the incubator was treated with Trypsin solution for 3 to 6 mins according to the days of ageing and then was stained by Giemsa solution.

\section{RESULT}

With the help of digital camera photographs of various chromosomal spread were taken from each sample under oil immersion lens. Later, the individual chromosomes are cut out, arranged in pairs thus karyotyping was done.

The numerical chromosomal evaluation of 20 mentally retarded children was done out of which $10 \%$ was observed having positive finding revealing abnormal Karyotype, the rest $90 \%$ karyotypes were found to be normal. Of the established numerical chromosomal abnormalities, 2 patients were found to have numerical Autosomal abnormalities giving rise to frequency of about $10 \%$ while no patient had numerical sex chromosomal abnormality. In this abnormal Karyotype the trisomy of $21^{\text {st }}$ pair of chromosome was found. These abnormal karyotypes are of children who are suffering from Down's syndrome.

Chi-square test is applied to find significance of sex in numerical chromosomal abnormality. According to yate's correction the numerical chromosomal abnormalities in relation with sex is highly insignificant. It was concluded that higher frequency of abnormal karyotypes in male patients is because in the present study $85 \%$ male patients and only $15 \%$ females were evaluated. 
Tables and charts

Table-01: Comparison of sex according to age group in mentally retarded children

\begin{tabular}{|c|c|c|}
\hline \multirow{2}{*}{$\boldsymbol{A G E}$ GROUP } & \multicolumn{2}{|c|}{$\boldsymbol{S E \boldsymbol { X }}$} \\
\cline { 2 - 3 } & MALE & FEMALE \\
\hline 1-4 Years & 0 & 1 \\
\hline 5-8 Years & 3 & 0 \\
\hline 9-12 Years & 9 & 0 \\
\hline 13-16 Years & 5 & 2 \\
\hline
\end{tabular}

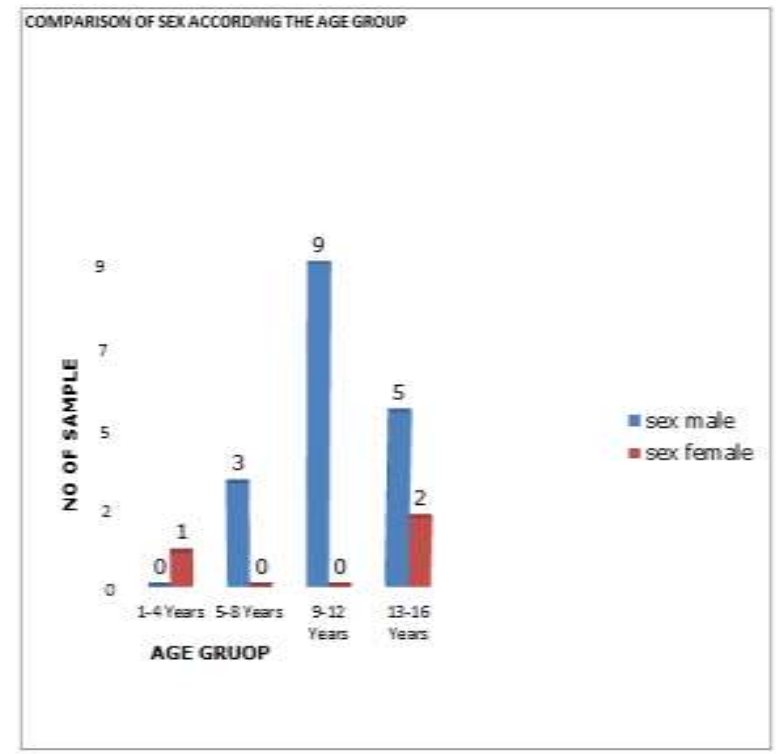

Table-2: The numerically abnormal karyotype in mentally retarded children

\begin{tabular}{|c|c|c|}
\hline NO OF SAMPLE & \multicolumn{2}{|c|}{ KARYOTYPE } \\
\cline { 2 - 3 } & NORMAL & ABNORMAL \\
\hline 20 & 18 & 2 \\
\hline
\end{tabular}

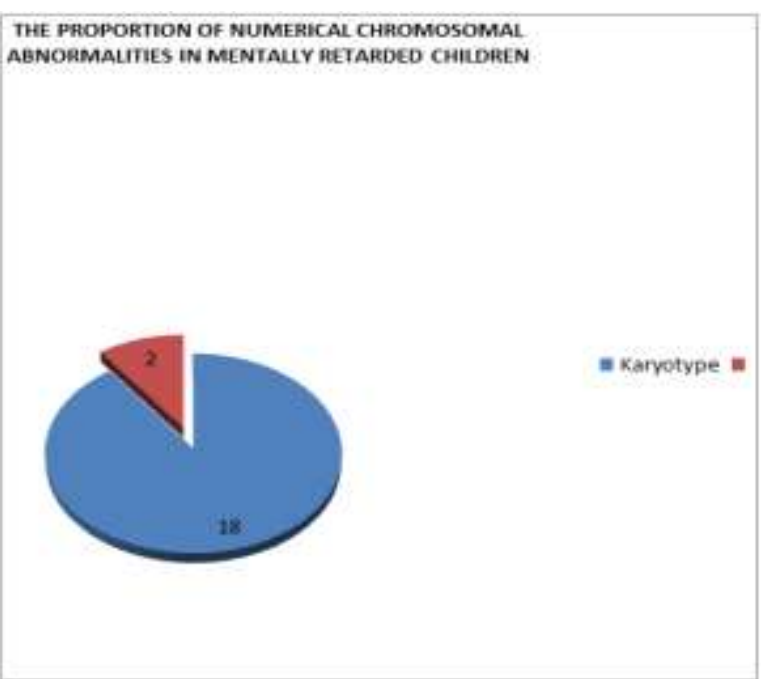

Table-03 : Comparison of karyotype according to age group

\begin{tabular}{|l|c|c|}
\hline \multirow{2}{*}{$A G E$ GROUP } & \multicolumn{2}{|c|}{ KARYOTYPE } \\
\cline { 2 - 3 } & NORMAL & ABNORMAL \\
\hline 1-4 Years & 1 & 0 \\
\hline 5-8 Years & 3 & 0 \\
\hline 9-12 Years & 8 & 1 \\
\hline 13-16 Years & 6 & 1 \\
\hline
\end{tabular}




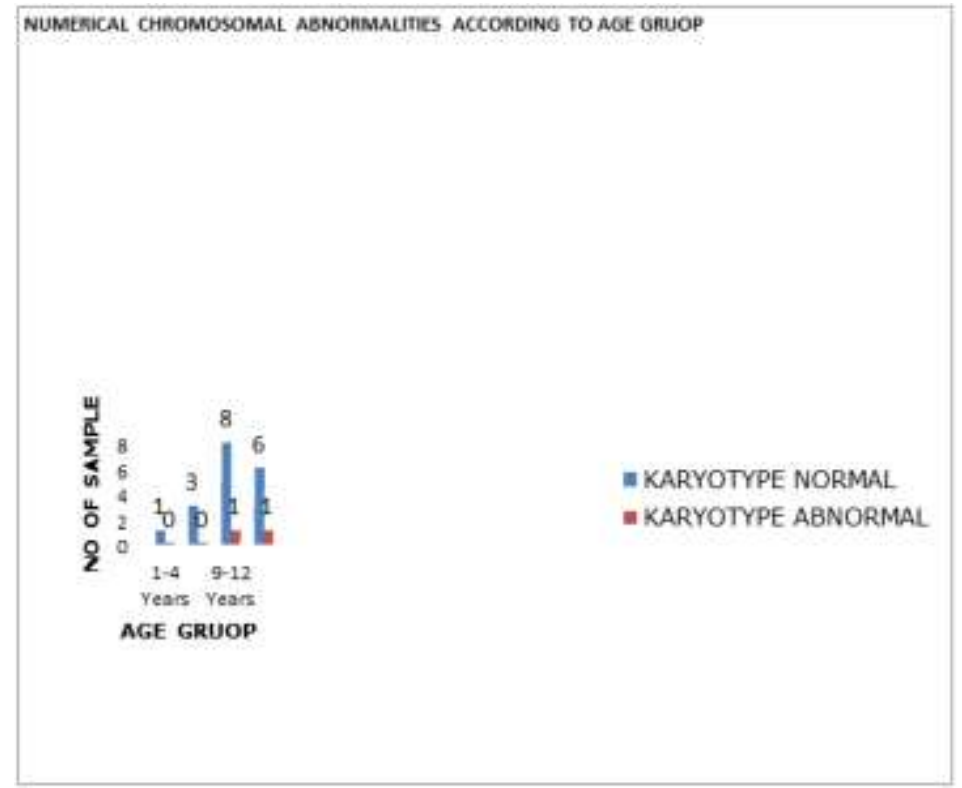

Table-04: Comparison of karyotype according to sex

\begin{tabular}{|l|l|l|l|}
\hline Sex & \multirow{2}{*}{ Number of Sample } & \multicolumn{2}{|c|}{ Karyotype } \\
\cline { 3 - 4 } & & Normal & Abnormal \\
\hline Male & 17 & 15 & 2 \\
\hline Female & 3 & 3 & 0 \\
\hline
\end{tabular}

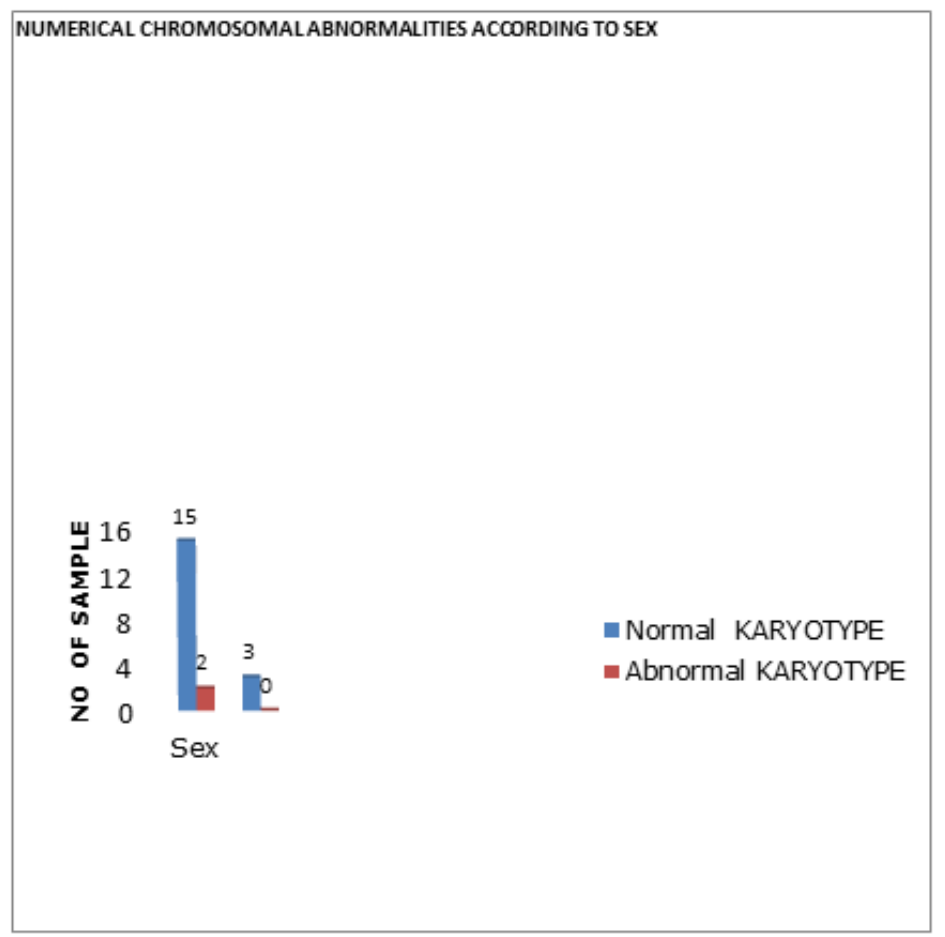

\section{DISCUSSION}

Genetics has an impact on the health of all individuals, their offspring and future generation. A number of surveys have indicated that globally at least 6-7 per 1000 persons have chromosomal abnormalities. 6 chromosomal abnormalities are thought to be the most common cause of Mental Retardation. However, apart from few selected types with typical aneuploidy, like Down's syndrome, klinefelter's syndrome,
Turner's syndrome etc. the frequency of detectable chromosomal abnormalities with idiopathic mental retardation is very low.

The relationship between gender and detection frequency of numerical anomalies is reported by a total 15 studies on 6601 persons, which provides sufficient data to draw conclusions. In total, a slight male predominance is reported (M: $\mathrm{F}=7.2 \%: 5.6 \%$ ) [7]. 
Cytogenic studies in the evaluation of children with mental retardation are to be expected in all children for whom the etiology of mental retardation is unknown. The reported frequency of chromosome anomalies detected by high resolution karyotyping (i.e. 650 bands) in patients evaluated for mental retardation varies between $9 \%$ and $36 \%$ [12]. The present study was performed using GTG banding technique and the frequency of chromosomal analysis and that to numerical is found $10 \%$. In the study conducted by Akeel A. Yaseen[9], the frequency of chromosomal abnormalities were found for around $67 \%$, which is very much high as compared to range between say $9 \%$ to $36 \%$ as results of several surveys, the reason behind it is the only included patients with severe mental retardation and hence concluded that the frequency of chromosomal abnormalities increased with the severity of mental retardation.

In the present study the selection criteria was the confirmation of mental retardation and not the criteria of the severity of mental retardation. So that children with all types of severity of mental retardation (from mild to profound) was included and the result observed was frequency of numerical chromosomal abnormalities is 10\%. Further Akeel A. Yaseen [9] added that his investigation showed sex chromosomal abnormalities less frequent than autosomal chromosomal abnormalities that account for $5 \%$ while the autosomal comprise of $62 \%$ of all 21 mentally retarded patients. These percentages seem to be in full agreement with those reported by other investigators. It is generally agreed that sex chromosome abnormalities are associated with slight decrease in the IQ and have generally less devastating effects than that of autosomal abnormalities. In comparison with present study $10 \%$ of autosomal abnormalities found while not single positive findings related to sex chromosomal abnormalities.

The authors van Karnebeek et al. found that there was a relationship between the number of minor anomalies and the likelihood of a chromosomal abnormality: a higher number of anomalies (more than 6) indicated a significantly higher likelihood to find a chromosomal abnormality. They concluded that all patients with no known cause for the Mental Retardation should have chromosome analysis performed. Van Karnebeek[7] found that approximately $10 \%$ of all patients with Mental Retardation had a chromosomal abnormality and recommended routine karyotyping in all patients for whom the aetiology of the Mental Retardation was unknown. In our study the children with mental retardation we observed $10 \%$ chromosomal abnormalities among 20 subjects.

Shiune and Lin [10] observed $22.43 \%$ of chromosomal abnormalities in the mentally retarded school children and stated that chromosomal abnormalities occurred in a high proportion of mentally retarded school children from southern Taiwan, with Trisomy 21 being the most prevalent. The findings indicated the need for increased attention to prenatal, perinatal and postnatal screening. The present study suggests the numerically abnormal Karyotype among 20 subjects is 02 , which both the abnormal Karyotype reveals Trisomy in the $21^{\text {st }}$ pair of chromosome. A cytogenic survey or all 588 patients in Strathmont Training Centre, an Australian hospital for the mentally retarded, was carried out by Sutherland and his colleagues. Abnormal karyotypes were found in 90(15.3\%) patients, of whom $73(12.4 \%)$ had clinical Down syndrome, $12(2.04 \%)$ other autosomal abnormalities, and 5(0.85\%) sex chromosome abnormalities. In comparison with present study cytogenic analysis was performed on 20 mentally retarded, and the abnormal karyotype was found in $10 \%$ patients, both children are suffering from Down's syndrome.

Genetic analysis of 169 mentally retarded (MR) children from Madras was carried out by B .R. Krishnan and A Ramesh and revealed chromosomal abnormalities in $17 \%$. Down syndrome was the major chromosomal anomaly $(24 / 169=14.2 \%)$. These included three cases of trisomy 21 mosaics and one case of de novo Robertsonian translocation. They stated that MR children with chromosomal abnormalities were either mildly or moderately retarded. Syndromes with known aetiology occurred in 3\% of the MR cases. Microcephaly, neonatal anoxia, perinatal stress and pharmacological attempt for abortion were found to be important pathogenic factors associated with MR. Birth order effects were found only among Down syndrome patients. Segregation analysis of the three groups of proband families (viz. mild, moderate and severe MR), indicated that autosomal recessive mode of inheritance is compatible in moderate and severe MR proband families.

In comparison with the present study cytogenic studies of mentally retarded children within the age group of 1 and $16 \mathrm{yrs}$. was performed with confirmation of mental retardation was and the criteria of the severity of mentally retarded was not focused. In all patients the chromosomal evaluation was performed with Giemsa banding. $10 \%$ of the observed karyotypes were found to be abnormal. In the abnormal karyotypes trisomy in the $21^{\text {st }}$ pair of chromosome was observed with no abnormality in the sex chromosomes.

\section{CONCLUSION}

We observed $10 \%$ karyotypes with the numerical chromosomal abnormalities and that is trisomy in the 21 st pair of chromosomes. Chi-square test is applied to find significance of sex in neumerical chromosomal abnormality. According to Yete's correction the neumerical chromosomal abnormalities in relation to sex is highly insignificant. We concluded that higher frequency of abnormal karyotypes in male 
patients is because in present study we have evaluated karyotypes of $85 \%$ male patients and $15 \%$ were females.

\section{ACKNOWLEDGEMENT}

Warm disposition untiring ever pleasant attitude, vast knowledge and experience of my esteemed teacher, mentor and elite guide Dr. S. S. Saiyad, M.S. (Anatomy), have played a key role in the course of my study.

Encouragement and moral support of my husband, Dr. Amit Divecha was indispensable, and of course my ever inspiring kids STASHA and RIYANSH, last but not the least my thanks to my parents and my in laws.

\section{REFERENCES}

1. Faed, M. J. W., Robertson, I., Field, M. A. S., \& Mellon, J. P. (1979). A chromosome survey of a hospital for the mentally subnormal. Clinical genetics, 16(3), 191-204.

2. Van Karnebeek, C. D. M., Scheper, F. Y., Abeling, N. G., Alders, M., Barth, P. G., Hoovers, J. M., \& Hennekam, R. C. (2002). Aetiology of mental retardation or borderline cognitive delay in 281 children referred to a tertiary care center: a prospective study. Mental retardation. Diagnostic studies on aetiology. Amsterdam, Netherlands: University of Amsterdam, 75-108.

3. Shevell, M. I., Ashwal, S., Donley, D., Flint, J., Gingold, M., Hirtz, D., ... \& Sheth, R. D. (2003). Practice parameter: evaluation of the child with global developmental delay: report of the Quality Standards Subcommittee of the American
Academy of Neurology and The Practice Committee of the Child Neurology Society. Neurology, 60(3), 367-380.

4. Knight, S. J., Regan, R., Nicod, A., Horsley, S. W., Kearney, L., Homfray, T., \& Flint, J. (1999). Subtle chromosomal rearrangements in children with unexplained mental retardation. The Lancet, 354(9191), 1676-1681.

5. Dutta, S., Shaw, J., Sinha, S., \& Mukhopadhyay, K. (2009). Chromosomal abnormalities associated with mental retardation in female subjects. Indian journal of human genetics, 15(1), 28.

6. Abdel Meguid, N., Zaki, M. S., \& Hammad, S. A. (2000). Premarital genetic investigations: effect of genetic counselling. EMHJ-Eastern Mediterranean Health Journal, 6 (4), 652-660, 2000.

7. Van Karnebeek, C. D., Jansweijer, M. C., Leenders, A. G., Offringa, M., \& Hennekam, R. C. (2005). Diagnostic investigations in individuals with mental retardation: a systematic literature review of their usefulness. European journal of human genetics, 13(1), 6-25.

8. Pal, G. P., \& Routal, R. V. (1986). A study of sutural bones in different morphological forms of skulls. Anthropologischer Anzeiger, 169-173.

9. Yasseen, A. A., \& Al-Musawi, T. A. (2001). Cytogenetics study in severely mentally retarded patients. Saudi medical journal, 22(5), 444-449.

10. Shiue, C. N., Lin, Y. H., Kuan, L. C., Lii, L. M., Tsai, W. H., Chen, Y. J., ... \& Kuo, P. L. (2004). Cytogenetic surveillance of mentally-retarded school children in southern Taiwan. Journal of the Formosan Medical Association= Taiwan yi zhi, 103(3), 218-224. 